\title{
The Physics of Electronic and Atomic Collisions
}

Lasers and other experimental tools such as storage rings for highly-charged ions, cluster beams, narrow-band synchrotron radiation and multi-detection devices are ensuring continuous progress in the field of electronic and atomic collisions. This was well documented by many invited and contributed papers at last year's 18th International Conference on the Physics of Electronic and Atomic Collisions illustrating the rôle played by lasers. Lasers are now used to prepare beams of polarized excited atoms, ions, and molecules, to detect atomic and molecular reaction products with unprecedented selectivity and sensitivity, and to study multiphoton and strong-field effects in ionization and detachment.

The European Research Conference in Physics - Electronic and Atomic Collisions: Processes at Low and Ultralow Energies (Giens, France; 10-15 September 1994) will highlight the trend towards exploiting new techniques by considering in greater detail collisions between: electrons and molecules; atoms, molecules and ions in beams, traps and low-temperature reactors; electrons and ions in merged beams and in storage beams; laser-cooled ions. But one should not forget that the field of electronic and atomic collisions spills over into many other areas. The four plenary lectures presented at the 18th ICPEAC gave surveys of important subfields. Ben Mottelson (Copenhagen) addressed "Shell effects in dusters". Clusters of atoms and molecules function as a bridge between the gas phase and the solid state and are actively studied using spectroscopic and collisional methods. Referring mainly to recent experimental results on shell effects in metal clusters, Mottelson related the "bunchiness" of the single-particle spectrum to shell structure and pointed out analogies to nuclear shell effects. Fritz Bosch (GSI Darmstadt) highlighted the impressive advances in and with ion-storage rings recently achieved at several facilities, notably ESR (GSI Darmstadt), TSR (Heidelberg), ASTRID (Aarhus), and CRYRING (Stockholm). In his lecture on "Atomic physics using storage cooler rings" he showed how electron and laser cooling of heavy ions leads to brilliant monochromatic beams of highly-charged ions which can be used for new kinds of precision experiments in atomic physics, both in spectroscopy and collisions. Many new results have already been obtained, extending from radiative and dielectronic recombination (see figure) to

\section{ERC}

\section{Electronic \& Atomic Collisions}

The European Research Conference in Physics - Electronic and Atomic Collisions: Processes at Low and Ultralow Energies (Giens, France; 10-15 September 1994) is organized by the European Science Foundation in association with the Electronic and Atomic Collisions Section of the EPS Atomic and Molecular Physics Division, with H. Hotop, the Section Chair, acting as the Conference Chair (participation limited to 100). For information, contact: J. Hendekovic, ESF, 1, quai Lezay-Marnésia, F-67080 Strasbourg Cedex \{tel.: +33 (-) 8876 7135 , fax: $+33(-) 88366987\}$.

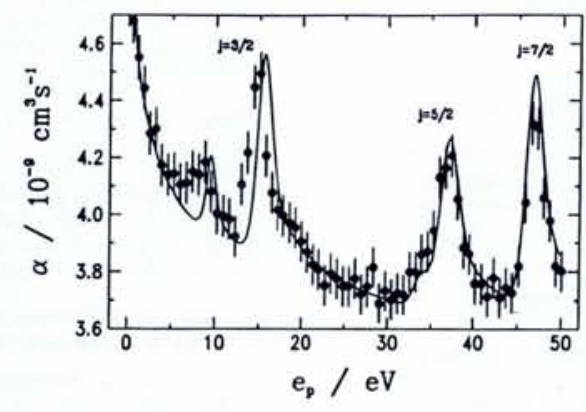

Sub-eV level structures of Li-like heavy ions. The excited states of highly charged heavy ions can be populated by recombination between intense beams of the ions stored in a ring and cold electrons. The recombination leads to characteristic K-radiation via cascades. By detuning the merged electron beam with respect to the ion beam, the recombination rate can be measured as a function of the relative velocity between ions and electrons. The rate decreases with increasing relative velocity (detuning), with peaks associated with sharply accelerated recombination owing to dielectronic recombination resonances at welldefined detuning energies. The resonance positions can be determined to high accuracy to allow the structure of heavy few-electron systems to be tested. The figure shows the recombination rate for Li-like $\mathrm{Au}^{76^{+}}$ions, as a function of the centre-of-mass detuning energy, as measured at the SIS/ESR complex at GSI, Darmstadt (the continuous line is a theoretical curve). Figure courtesy of F. Bosch, GSI, Darmstadt.

electron capture, $\mathrm{x}$-ray spectroscopy, tests of relativity, and atomic astrophysics.

The third plenary lecture was devoted to the theory of electron collisions with atoms, ions and molecules. Phil Burke (Belfast), one of the leaders in this field, demonstrated the continuous advances in calculating elastic and inelastic processes (including ionization) in electron-atom and electron-ion collisions which are important in gaseous discharges, fusion plasmas and astrophysics. Substantial progress has been made in the treatment of relativistic effects for heavier systems and in spin-dependent scattering. Electron-molecule scattering theory successfully tackles the problems associated with electronically excited final states and the rôle of nuclear motion and non-adiabatic effects in dissociative and vibrational excitation. Spin-dependent effects in electron-molecule scattering present a challenge for the future.

Kate Kirby (Cambridge, USA) reviewed "Atmospheric physics, collision physics, and global change", demonstrating that studies of electronic and atomic collisions can help provide partial answers in developing more reliable models of the atmosphere. Theory presently cannot predict the amount of ozone ("missing ozone problem"), and pathways for increased ozone production are being investigated. One of the great challenges in atmospheric modelling is to predict the influence of clouds.

H. Hotop, Kaiserslautern 18th ICPEAC The 18th International Conference on the Physics of Electronic and Atomic Collisions (ICPEAC) represents a biennial series of conferences dedicated to the fields of electronic and atomic collisions and related areas of atomic and molecular physics. In particular, it deals with the interactions between atoms, ions, molecules, electrons, positrons, and photons. The 18th ICPEAC (21-27 July 1993) took place in the beautiful and well-suited Concert Hall in Aarhus and was organized in an excellent way by members of the Institute of Physics, Aarhus, with Torkild Andersen and Bent Fastnup serving as Local Co-Chairs. There were about 730 participants from 42 countries, of which about 200 were students or postdocs. The programme included 4 plenary lectures 26 reviews, 36 progress reports, and 10 oral presentations of selected contributed papers (hot topics). The abstract booklet contains 845 contributed papers which were presented as posters during the afternoons in the Ridehuset. Both the lectures and the poster sessions testified that the field represents a lively and innovative area of research. The proceedings (ISBN 1-56396-290-X) were published by the American Institute of Physics and are available from AIP Conference Proceedings, AIP, 500 Sunnyside Blvd., Woodbury, NY 11797 2999, USA. The 20th ICPEAC will be held in Whistler, BC, Canada (26 July - 1 August 1995 contact: Int. Conf. Services, 604-850 West Hastings St., Vancouver, BC, V6C 1E1, Canada tel.: +1-604-681 2153 ; fax: +-604-681 10 49) and the 21th ICPEAC in Vienna (July/August 1997; contact: H. Winter, Inst. f. Allgemeine Physik, TU Wien, Wiedner Haupstrasse 8-10, A-1040 Vienna; tel.: +43-1-588 01; fax: +43-1-56 42 03; winter @ eapv38.tuwein.ac.at).

\section{The Faculty of Science of the University of Lausanne has an opening for a full time}

\section{Professorship in Physics of Living Matter}

This field is not yet represented in our University. The candidate should be an experimental physicist with specialisation in the physics of living matter. He/she will set up a new research group and establish collaboration with groups in existing related fields, particularly, for example, with those in the Department of Biology, the Medical Faculty, the Swiss Cancer Research Institute, the Physics Department of the Swiss Federal Institute of Technology (EPFL) and with other neighbouring universities.

$\mathrm{He} /$ she will participate in the teaching (in French) of general physics to students in science and give advanced physics students in physics or physical engineers an optional course about the physics of living matter. These lectures will also be open to biology, chemistry and medical students. Lectures will be given in French.

Applications (C.V., list of publications, reprints of the three most significant publications, research projects and three references) should be sent before October 15, 1994 to the Dean of the Faculty of Science, $\mathrm{CP}, \mathrm{CH}-1015$ Lausanne. For further information:

Prof. W.-D. Schneider, tel. +4121/6923661 or 69236 60, Email: wschneid@ipe.unil.ch. For administrative matters: phone $+4121 / 6923501$, fax $+4121 / 6923505$. 